\title{
Toward Computing Linguistic Fuzzy Graphs And Applying to Illegal Immigration Problem
}

\author{
Nguyen Van Han*, Phan Cong Vinh \\ Faculty of Information Technology, Nguyen Tat Thanh University. 300A Nguyen Tat Thanh street, Ward 13, \\ District 4, Ho Chi Minh city, Vietnam. \\ Email:\{nvhan,pcvinh\}@ntt.edu.vn
}

\section{Abstract}

In this paper, we study linguistic fuzzy graph properties which consist of fuzzy paths, cut vertex and bridge.

We use hedge algebra and linguistic variables for modeling to reduce complexity in computation. Modeling the Illegal immigration problem is also introduced

\begin{abstract}
Received on 01 September 2020; accepted on 19 September 2020; published on 24 September 2020
Keywords: Fuzzy set, Fuzzy logic, Linguistic variable, Hedge algebra, Fuzzy graph

Copyright (C) 2020 Nguyen Van Han et al., licensed to EAI. This is an open access article distributed under the terms of the Creative Commons Attribution license (http: / / creativecommons . org/licenses/by/3.0/), which permits unlimited use, distribution and reproduction in any medium so long as the original work is properly cited.
\end{abstract}

doi:10.4108/eai.24-9-2020.166361

\section{Introduction}

In everyday life, people use natural language (NL) for analyzing, reasoning, and finally, make their decisions. Computing with words (CWW) [9] is a mathematical solution of computational problems stated in an NL. CWW based on fuzzy set and fuzzy logic, introduced by L. A. Zadeh is an approximate method on interval $[0,1]$. In linguistic domain, linguistic hedges play an important role for generating set of linguistic variables. A well known application of fuzzy set is fuzzy graph $[1,5-8]$, combined fuzzy set with graph theory. Fuzzy graph $(\mathbb{F} \mathbb{G})$ has a lots of applications in both modeling and reasoning fuzzy knowledge such as Human trafficking, internet routing, illegal immigration [3] on interval $[0,1]$ but not in linguistic values, However, many applications cannot model in numerical domain [9], for example, linguistic summarization problems [10]. To solve this problem, in the paper, we use an abstract algebra, called hedge algebra $(\mathbb{H} \mathbb{A})$ as a tool for computing with words. The remainder of paper is organized as follows. Section 2 reviews some main concepts of computing with words based on $\mathbb{H} \mathbb{A}$. Important section 3 studies a linguistic fuzzy graph modeling with words using $\mathbb{H} \mathbb{A}$ and its properties. Section 4 presents an application of $\mathbb{L} \mathbb{G}$. Section 5 outlines conclusions and future work.

*Corresponding author. Email: nvhan@ntt.edu.vn

\section{Preliminaries}

This section presents basic concepts of $\mathbb{H} \mathbb{A}$ and some important knowledge used in the paper.

\section{Hedge algebra}

In this section, we review some $\mathbb{H} \mathbb{A}$ knowledges related to our research paper and give basic definitions. First definition of a $\mathbb{H} \mathbb{A}$ is specified by 3-Tuple $\mathbb{H} \mathbb{A}=$ $(X, H, \leq)$ in [11]. In [12] to easily simulate fuzzy knowledge, two terms $G$ and $C$ are inserted to 3-Tuple so $\mathbb{H} \mathbb{A}=(X, G, C, H, \leq)$ where $H \neq \emptyset, G=\left\{c^{+}, c^{-}\right\}, C=$ $\{0, W, 1\}$. Domain of $X$ is $\mathbb{L}=\operatorname{Dom}(X)=\{\delta c \mid c \in G, \delta \in$ $H^{*}$ (hedge string over $\left.\left.\mathrm{H}\right)\right\},\{\mathbb{L}, \leq\}$ is a POSET (partial order set) and $x=h_{n} h_{n-1} \ldots h_{1} c$ is said to be a canonical string of linguistic variable $x$.

Example 1. Fuzzy subset $X$ is Age, $G=\left\{c^{+}=\right.$ young; $c^{-}=$old $\}, H=\{$ less; more; very $\}$ so term-set of linguistic variable Age $\mathrm{X}$ is $\mathbb{L}(\mathrm{X})$ or $\mathbb{L}$ for short:

$\mathbb{L}=\{$ very less young ; less young ; young ; more young ; very young ; very very young...\}

Fuzziness properties of elements in $\mathbb{H} \mathbb{A}$, specified by $f m$ (fuzziness measure) [12] as follows:

Definition 2.1. A mapping $f m: \mathbb{L} \rightarrow[0,1]$ is said to be the fuzziness measure of $\mathbb{L}$ if:

1. $\sum_{c \in\left\{c^{+}, c^{-}\right\}} f m(c)=1, f m(0)=f m(w)=f m(1)=0$.

2. $\sum_{h_{i} \in H} f m\left(h_{i} x\right)=f m(x), \quad x=h_{n} h_{n-1} \ldots h_{1} c$, the canonical form. 


$$
\text { 3. } f m\left(h_{n} h_{n-1} \ldots h_{1} c\right)=\prod_{i=1}^{n} f m\left(h_{i}\right) \times \mu(x) \text {. }
$$

The Truth and meaning are fundamental important concepts in fuzzy logic, artificial intelligence and machine learning. In RCT (restriction-centered theory) in [9], truth values are organized as a hierarchy with ground level or first-order and secondorder. First order truth values are numerical values whereas second order ones are linguistic truth values. A linguistic truth value, say $\ell$, is a fuzzy set. We study linguistic truth values on POSET $\mathbb{L}$ whose elements are comparable [15].

Definition 2.2. A โ STRUCT $[\rho]$ on relational signature $\rho$ is a tuple:

$$
\overleftarrow{L}=\left\langle\mathbb{L}, f_{a_{i}}^{\complement}, c_{j}^{\complement}\right\rangle
$$

Consists of a universe $\mathbb{L} \neq \emptyset$ together with an interpretation of:

- each constant symbol $c_{j}$ from $\rho$ as an element $c_{j}^{\Gamma_{0}} \in \mathbb{L}$

- each $a_{i}$-ary function symbol $f_{a_{i}}$ from $\rho$ as a function:

$$
f_{i}^{\complement}: \mathbb{L}^{a_{i}} \rightarrow \mathbb{L}
$$

In $\mathbb{H} \mathbb{A}, \ell \in \mathbb{L}$ and there are order properties:

Theorem 2.1.in [11] let $\ell_{1}=h_{n} \ldots h_{1} u$ and $\ell_{2}=$ $k_{m} \ldots k_{1} u$ be two arbitrary canonical representations of $\ell_{1}$ and $\ell_{2}$, then there exists an index $j \leq \bigwedge\{m, n\}+1$ such that $h_{i}=k_{j}$, for $\forall i<j$, and:

1. $\ell_{1}<\ell_{2}$ iff $h_{j} x_{j}<k_{j} x_{j}$ where $x_{j}=h_{j-1} \ldots h_{1} u$;

2. $\ell_{1}=\ell_{2}$ iff $m=n=j$ and $h_{j} x_{j}=k_{j} x_{j}$;

3. $\ell_{1}$ and $\ell_{2}$ are incomparable iff $h_{j} x_{j}$ and $k_{j} x_{j}$ are incomparable;

Example 2. Consider linguistic variables: $\{\mathscr{V}$ true, $\mathscr{P}$ true, $\mathscr{L}$ true $\} \in \mathbb{H}$, in which $\{\mathscr{V}$ true, $\mathscr{P}$ true, $\mathscr{L}$ true $\}$ stand for : very true, possible true and less true are linguistic truth values generated from variable truth. Assume propositions $\mathrm{p}=$ "Lucie is young is $\mathscr{V}$ true" and q = "Lucie is smart is $\mathscr{P}$ true", interpretations on $\mathfrak{H}$ are:

- $\operatorname{truth}(p)=\mathscr{V}$ true $\in \mathbb{H}$, truth is a unary function.

- $p \wedge q=\mathscr{V}$ true $\wedge \mathscr{P}$ true $=\mathscr{P}$ true $\in \mathbb{H} . \wedge$ is $\quad$ a binary function.

- $p \vee q=\mathscr{V}$ true $\vee \mathscr{P}$ true $=\mathscr{V}$ true $\in \mathbb{H} . \quad \vee \quad$ is $\quad$ a binary function.

\section{$3 \quad$ Fuzzy graph model based on linguistic variables}

The first $\mathbb{F G}$ (fuzzy graph) was introduced in [1], which vetices and edges's values are in unit interval $[0,1]$. Many $\mathbb{F} \mathbb{G}^{\prime}$ 's theories were developed in $[2,3]$ in which computational phases have a bit complex because converting from linguistic to number value to compute. To reduce complexity, we directly compute by applying computing with word method [9]

\section{Our graph model}

Our fuzzy graph is called $\mathbb{L} \mathbb{G}$ (linguistic graph) with $\mathbb{L}$ is domain of both vertex $\mathbb{V}$ and $\mathbb{E}$, see Fig. 2.

Definition 3.1. A linguistic graph $\mathbb{L} \mathbb{G}=(\mathbb{V}, \rho, \delta)$ consist of set $\mathbb{V}$, a fuzzy vertex set $\rho$ on $\mathbb{V}$ and a fuzzy edge set $\delta$ on $\mathbb{V}$ so that $\delta(u, v) \leq \rho(u) \wedge \rho(v)$ for every $u, v \in \mathbb{V}$.

$$
\mathbb{L} \mathbb{G}=\{(\mathbb{V}, \rho, \delta): \rho \widetilde{\subset} \mathbb{V} ; \delta \widetilde{\subset} \mathbb{E}\}
$$

On any graph, it always have paths, cut vertices and bridge edges. Let $u \stackrel{\mathscr{D}}{\rightarrow} v$ be a path between two vertices $u$ and $v$

Definition 3.2. 1. A path $\mathscr{P}$ of length $n$ in a fuzzy graph $\mathbb{L} \mathbb{G}$ is a sequence of distinct vertices $v_{1}, v_{2}, \ldots, v_{n}$ with condition is $\delta\left(v_{i}, v_{j}\right) \in \mathbb{L} ; i=$ $\overline{1, n}$

2. The connecting strength between $u, v \in \mathbb{V}$, denoted by $\operatorname{Conn}_{\mathbb{L} \mathbb{G}}(u, v)$, is the maximum of the strength of all paths between $u$ and $v, u \stackrel{\mathscr{P}}{\sim} v$.

3. An edge $e \in \mathbb{E}$ is called fuzzy bridge if deleting $e$ from $\mathbb{L} \mathbb{G}$ reduces the strength between some pair of vertices.

$$
\operatorname{Conn}_{\mathbb{L} \mathbb{G}-e}(u, v)<\operatorname{Conn}_{\mathbb{L} \mathbb{G}}(u, v)
$$

4. An vertex $w \in \mathbb{V}$ is called fuzzy cut vertex if deleting $w$ and adjacent edges to (or from $w$ ) from $\mathbb{L} \mathbb{G}$ reduces the strength between some pair of vertices.

$$
\operatorname{Conn}_{\mathbb{L} \mathbb{G}-w}(u, v)<\operatorname{Conn}_{\mathbb{L} \mathbb{G}}(u, v)
$$

$\mathbb{L} \mathbb{G}$ is the special case of $\mathbb{F} \mathbb{G}$ on linguistic domain $\mathbb{L}$ so it have some common and separate properties. Immediately from Definition 3.2 we infer the following important property on $\mathbb{L} \mathbb{G}$

Property 3.1. For every vertices $u$ and $v$ on linguistic graph $\mathbb{L} \mathbb{G}$ the connection strength between them do not increase if we delete cut vertex or bridge edge. 


\section{An application of $\mathbb{L} \mathbb{G}$ in illegal immi- gration}

\subsection{Immigration problem}

Illegal immigration problem was introduced in [4]. People from Asia and Africa are seeking to enter the U. S. illegal over the Mexican border by six main routes as following:

$R_{1}:$ China $\rightarrow$ Columbia $\rightarrow$ Guatemala $\rightarrow$ Mexico $\rightarrow$ U.S.

$R_{2}:$ India $\rightarrow$ Guatemala $\rightarrow$ Mexico $\rightarrow$ U.S.

$R_{3}:$ Ethiopia $\rightarrow$ S.Africa $\rightarrow$ Brazil $\rightarrow$ Ecuador $\rightarrow$ Mexico $\rightarrow$ U.S.

$R_{4}:$ Somalia $\rightarrow U A E \rightarrow$ Russia $\rightarrow \mathrm{Cuba} \rightarrow$

Columbia $\rightarrow$ Mexico $\rightarrow$ U.S.

$R_{5}:$ Nigeria $\rightarrow$ Spain $\rightarrow$ Cuba $\rightarrow$ Columbia $\rightarrow$

Mexico $\rightarrow$ U.S.

$R_{6}:$ Nigeria $\rightarrow$ Spain $\rightarrow$ Columbia $\rightarrow$ Mexico $\rightarrow$ U.S.

The size of flow from country to country is reported in linguistic terms very low, low, medium, high, very high [3] models as data table in Fig. 1:

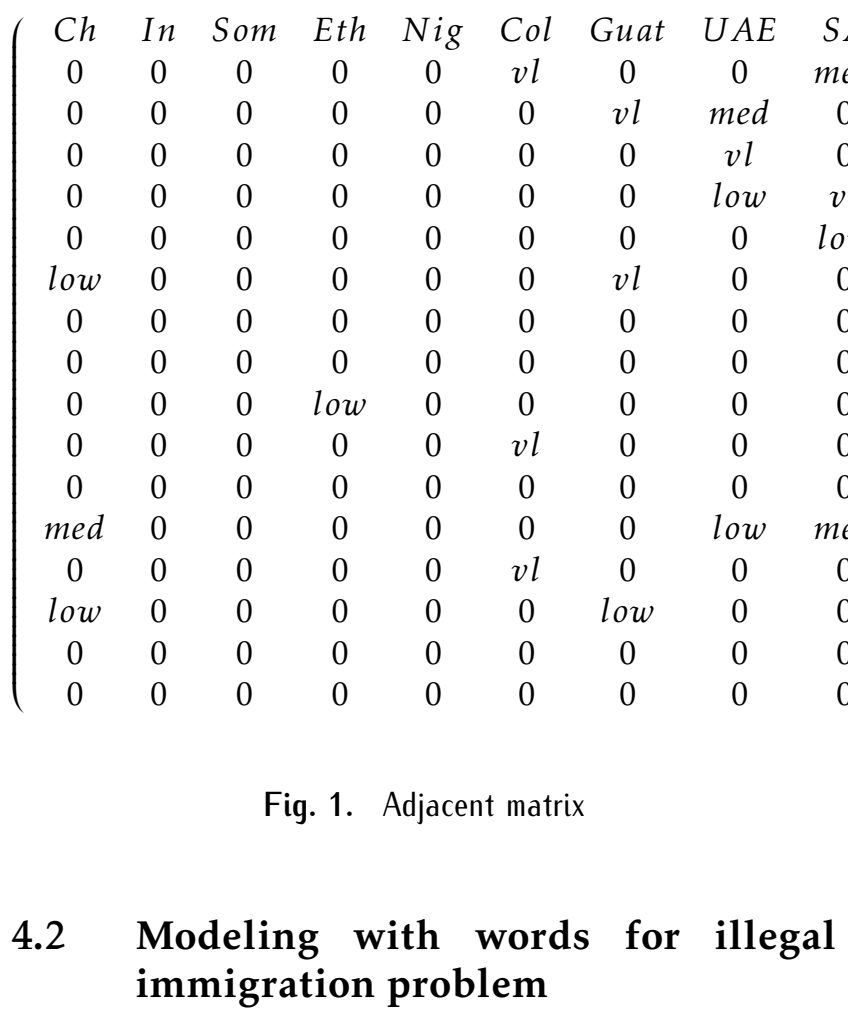

To model data table in Fig. 1 with linguistic variable in $\mathbb{L}$, use a $\mathbb{H} \mathbb{A}$ as: $\mathbb{H} \mathbb{A}=\left\{X, \mathcal{H},\left\{c^{+}, c^{-}\right\},\{0, W, 1\}, \leq\right.$ \} , in which $X=$ "size of flow", $c^{+}=$high, $c^{-}=$low, $W=$ "med", $\mathcal{H}=$ "very", vl = "very low". Using domain converting method in [13-15], the $\mathbb{L} \mathbb{G}$ is constructed as in Fig. 2 (some edges don't draw to keep clearing figure).

\subsection{Computing on $\mathbb{L} \mathbb{G}$}

Computing on $\mathbb{L} \mathbb{G}$ based on property 3.1 about cut vertices or bridge. Applying Theorem 2.1 by ordering $0<$ very low $<$ low $<W<$ high $<$ very high $<1$

Example 3. From Fig. 2: $\operatorname{Conn}_{\mathbb{L} \mathbb{G}-(\text { China,x })}($ China, $x) \leq$ Conn $_{\mathbb{L} \mathbb{G}}($ China, $x)$ on path China $\stackrel{\mathscr{P}}{\rightarrow} x, x \in \mathbb{V}$, for example $x=$ Russia then $\delta$ (China,Russia $)=$ low and $\operatorname{Conn}_{\mathbb{L} \mathbb{G}-(\text { China,Russia })}($ China, Russia $)=W \wedge v l \wedge l o w=$ $v l$, so China is the cut vertex. For controling people flow to U. S., we should delete China cut vertex and so on.

\section{Conclusions and future work}

We have introduced a fuzzy graph model which called $\mathbb{F} G$ with two advantages

1. Modeling fuzzy graph uses linguistic variable by applying hedge algebra

2. Computing with words on linguistic variable without converting to numeric therefore reducing number of operators for computation phases.

$\left.\begin{array}{ccccccc}\text { Sp } & \text { Br } & \text { Rus } & \text { Cuba } & \text { Ec } & \text { Mex } & \text { US } \\ \text { low } & 0 & \text { low } & 0 & 0 & 0 & \text { high } \\ 0 & 0 & 0 & 0 & 0 & 0 & \text { med } \\ 0 & 0 & 0 & 0 & 0 & 0 & \text { low } \\ 0 & 0 & 0 & 0 & 0 & 0 & \text { low } \\ \text { high } & 0 & 0 & 0 & 0 & 0 & \text { med } \\ \text { high } & 0 & 0 & 0 & 0 & v l & \text { med } \\ 0 & 0 & 0 & 0 & 0 & \text { med } & \text { med } \\ 0 & 0 & v l & 0 & 0 & 0 & 0 \\ 0 & v l & 0 & 0 & 0 & 0 & 0 \\ 0 & 0 & 0 & v l & 0 & 0 & 0 \\ 0 & 0 & l o w & 0 & v l & 0 & \text { med } \\ \text { high } & 0 & 0 & v l & 0 & \text { low } & \text { high } \\ \text { low } & 0 & 0 & 0 & 0 & 0 & \text { low } \\ 0 & 0 & 0 & 0 & 0 & \text { low } & \text { med } \\ \text { low } & 0 & 0 & 0 & 0 & 0 & \text { high } \\ 0 & 0 & 0 & 0 & 0 & 0 & 0\end{array}\right)$

Our next study is studying algorithms to construct and compute $\mathbb{L} \mathbb{G}=(\mathbb{V}, \rho, \delta)$ 


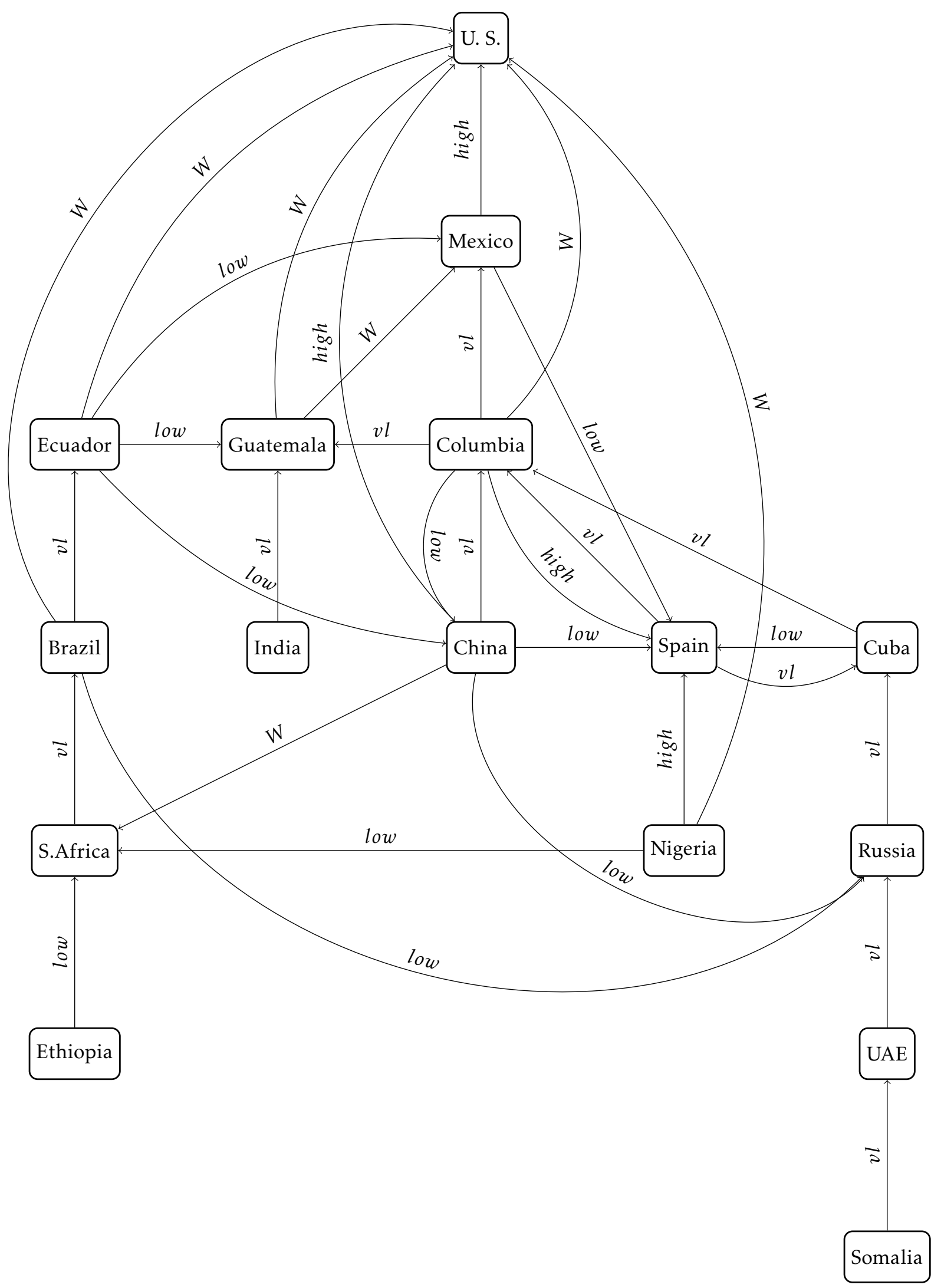

Fig. 2. $\mathbb{L} \mathbb{G}$ for Immigration problem 


\section{References}

[1] Rosenfeld, A, Fuzzy Graphs, Fuzzy Sets and Their Applications, pp. 77-95. Academic Press, New York (1975).

[2] J.N. Mordeson, P.S. Nair Fuzzy Graphs and Fuzzy Hypergraphs, Physica-Verlag, Heidelberg (2000)

[3] John N. Mordeson, Sunil Mathew, Advanced Topics in Fuzzy Graph Theory, Springer Nature Switzeland AG, 2019

[4] Routes to the U.S, Mapping Human Smuggling Networks, https://www.insightcrime.org/news/analysis/routesto-the-us-mapping-human-smuggling-networks/

[5] B.Kosko, Fuzzy Cognitive Maps, Internation Journal of Man-Machine Studies, 24: pp.65-75, 1986.

[6] Osonde A Osoba and B.Kosko, Fuzzy Cognitive Maps of Public Support for Insurgency and Terrorism, Journal of Defense Modeling and Simulation: Applications, Methodology, Vol. 14(I): 17-32, 2017.

[7] Michael Glykas, Fuzzy Cognitive Maps, Advances in Theory, Tools and Applications, Springer, 2010.

[8] Elpiniki I. Papageorgiou Fuzzy Cognitive Maps for Applied Science and Engineering From Fundamentals to Extensions and Learning Algorithms, Springer-Verlag Berlin Heidelberg 2014.

[9] L.A.Zadeh,Computing with Words - Principal Concepts and Ideas. Studies in Fuzziness and Soft Computing,
Springer 2012.

[10] J. Kacprzyk, A. Wilbik, and S. Zadrożny.:Linguistic Summarization of Trends: a Fuzzy Logic based Approach. The 11th International Conference Information Processing and Management of Uncertainty in Knowledgebased Systems, pp.2166-2172, 2006.

[11] Nguyen Cat Ho and W.Wechler, Hedge Algebras: An Algebraic Approach to Structure of Sets of Linguistic Truth Values, Fuzzy Sets and Systems 35(1990), 281-293

[12] Cat-Ho Nguyen, Nguyen Van Long, Fuzziness Measure on Complete Hedge Algebras and Quantifying Semantics of Terms in Linear Hedge Algebras. Fuzzy Sets and Systems 158(4): 452-471, 2007.

[13] Nguyen Van Han, Phan Cong Vinh. Toward Modeling and Reasoning with Words Based on Hedge Algebra, EAI Endorsed Trans. Context-aware Syst. \& Appl. 5(15): e5, 2018.

[14] Nguyen Van Han, Phan Cong Vinh. Modeling with Words Based on Hedge Algebra, 7th EAI International Conference, ICCASA 2018 and 4th EAI International Conference, ICTCC 2018, LNICST 266, pp: 211 - 217, Springer, 2019.

[15] Nguyen Van Han, Phan Cong Vinh (2020), Reasoning with Words: A Hedge Algebra Linguistic Cognitive Map Approach, Concurrency and Computation Practice and Experience. doi: $10.1002 /$ cpe. 5711 